\title{
MONOTONICITY AND LOGARITHMIC CONVEXITY RELATING TO THE VOLUME OF THE UNIT BALL
}

\author{
FENG QI AND BAI-NI GUO
}

\begin{abstract}
Let $\Omega_{n}$ stand for the volume of the unit ball in $\mathbb{R}^{n}$ for $n \in \mathbb{N}$. In the present paper, we prove that the sequence $\Omega_{n}^{1 /(n \ln n)}$ is logarithmically convex

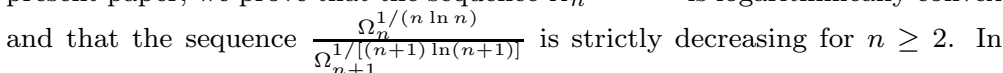
addition, some monotonic and concave properties of several functions relating to $\Omega_{n}$ are extended and generalized.
\end{abstract}

\section{INTRODUCTION}

In [5, Lemma 2.39], the following result was obtained: The function

$$
\frac{1}{x} \ln \Gamma\left(1+\frac{x}{2}\right)
$$

is strictly increasing from $[2, \infty)$ onto $[0, \infty)$ and

$$
\lim _{x \rightarrow \infty}\left[\frac{1}{x \ln x} \ln \Gamma\left(1+\frac{x}{2}\right)\right]=\frac{1}{2}
$$

where

$$
\Gamma(x)=\int_{0}^{\infty} t^{x-1} e^{-t} \mathrm{~d} t
$$

for $x>0$ denotes the classical Euler gamma function $\Gamma(x)$. From this, the following conclusions were deduced in [5, Lemma 2.40]: The sequence $\Omega_{n}^{1 / n}$ decreases strictly to 0 as $n \rightarrow \infty$, the series $\sum_{n=2}^{\infty} \Omega_{n}^{1 / \ln n}$ is convergent, and

$$
\lim _{n \rightarrow \infty} \Omega_{n}^{1 /(n \ln n)}=e^{-1 / 2}
$$

where

$$
\Omega_{n}=\frac{\pi^{n / 2}}{\Gamma(1+n / 2)}
$$

stands for the $n$-dimensional volume of the unit ball $\mathbb{B}^{n}$ in $\mathbb{R}^{n}$. Further, it was conjectured in [5, Remark 2.41] that the function in (2) is strictly increasing from $[2, \infty)$ onto $\left[0, \frac{1}{2}\right)$ and this would imply that the sequence $\Omega_{n}^{1 /(n \ln n)}$ is strictly decreasing for $n \geq 2$.

1991 Mathematics Subject Classification. Primary 33B15; Secondary 26 D07.

Key words and phrases. Monotonicity; logarithmical convexity; volume; unit ball; inequality; gamma function.

The first author was partially supported by the China Scholarship Council and the Science Foundation of Tianjin Polytechnic University.

This paper was typeset using $\mathcal{A M}_{\mathcal{M}} \mathcal{S}$-LATEX. 
In [3, Theorem 1.5], it was proved that the function

$$
\frac{\ln \Gamma(x+1)}{x \ln x}
$$

is strictly increasing from $(1, \infty)$ onto $(1-\gamma, 1)$, where $\gamma$ is the Euler-Mascheroni constant. From this, above-mentioned conjecture in [5, Remark 2.41] were resolved in [3, Corollary 3.1]. In addition, it was conjectured in [3, Conjecture 3.3] that the function (6) is concave on $(1, \infty)$.

In [9] and [13, Theorem 4], the function (6) was proved to be strictly increasing on $(0, \infty)$.

In [10, Section 3], the function (6) was proved to be concave for $x>1$.

In $[6$, Theorem 1.1], it was proved that

$$
(-1)^{n-1}\left[\frac{\ln \Gamma(x+1)}{x \ln x}\right]^{(n)}>0
$$

for $x>0$ and $n \in \mathbb{N}$. More strongly, the reciprocal of the function (6) was proved in $[6$, Theorem 1.4] to be a Stieltjes transform for $x \in \mathbb{C} \backslash(-\infty, 0]$, where $\mathbb{C}$ is the set of all complex numbers. Furthermore, among other things, it was directly shown in $[7$, Theorem 1.1] that the function (6) for $x \in \mathbb{C} \backslash(-\infty, 0]$ is a Pick function.

In $[2$, Lemma 4$]$, it was demonstrated that the function

$$
F(x)=\frac{\ln \Gamma(x+1)}{x \ln (2 x)}
$$

is strictly increasing on $[1, \infty)$ and strictly concave on $[46, \infty)$. With the help of this, the double inequality

$$
\exp \left(\frac{a}{n(\ln n)^{2}}\right) \leq \frac{\Omega_{n}^{1 /(n \ln n)}}{\Omega_{n+1}^{1 /[(n+1) \ln (n+1)]}}<\exp \left(\frac{b}{n(\ln n)^{2}}\right)
$$

was turned out in [2, Theorem 2] to be valid for $n \geq 2$ if and only if

$$
a \leq \ln 2 \ln \pi-\frac{2(\ln 2)^{2} \ln (4 \pi / 3)}{3 \ln 3}=0.3 \cdots \quad \text { and } \quad b \geq \frac{1+\ln (2 \pi)}{2}=1.4 \cdots .
$$

It is clear that the function in (2) is equivalent to (8). Therefore, the above conjecture posed in [5, Remark 2.41] was verified once again.

The first aim of this paper is to extend the ranges of $x$ such that the function $F(x)$ is both strictly increasing and strictly concave respectively as follows.

Theorem 1. On the interval $\left(0, \frac{1}{2}\right)$, the function $F(x)$ defined by (8) is strictly increasing; On the interval $\left(\frac{1}{2}, \infty\right)$, it is both strictly increasing and strictly concave.

The second aim of this paper is, with the aid of Theorem 1, to generalize the decreasing monotonicity of the sequence $\Omega_{n}^{1 /(n \ln n)}$ for $n \geq 2$, the conjecture in [5, Remark 2.41], and the main result in [3, Corollary 3.1], to the logarithmic convexity.

Theorem 2. The sequence $\Omega_{n}^{1 /(n \ln n)}$ is strictly logarithmically convex for $n \geq 2$. Consequently, the sequence

$$
\frac{\Omega_{n}^{1 /(n \ln n)}}{\Omega_{n+1}^{1 /[(n+1) \ln (n+1)]}}
$$

is strictly decreasing for $n \geq 2$. 
In [2, Lemma 3], the double inequality

$$
\frac{2}{3}<\left[1-\frac{\ln x}{\ln (x+1)}\right] x \ln x \triangleq G(x)<1
$$

was verified to be true for $x \geq 3$. The right-hand side inequality in (12) was also utilized in the proof of the inequality (9).

The third aim of this paper is to extend and generalize the inequality (12) to a monotonicity result as follows.

Theorem 3. The function $G(x)$ defined in the inequality (12) is strictly increasing on $(0, \infty)$ with

$$
\lim _{x \rightarrow 0^{+}} G(x)=-\infty \quad \text { and } \quad \lim _{x \rightarrow \infty} G(x)=1 .
$$

\section{REMARKS}

Before proving our theorems, we are about to give some remarks on them and the volume of the unit ball in $\mathbb{R}^{n}$.

Remark 1. It is obvious that Theorem 1 extends or generalizes the conjecture posed in [5, Remark 2.41] and the corresponding conclusions obtained in [3, Corollary 3.1] and $[2$, Lemma 4$]$ respectively.

Remark 2. For $a>1$ and $x>0$ with $x \neq \frac{1}{a}$, let

$$
F_{a}(x)=\frac{\ln \Gamma(x+1)}{x \ln (a x)} .
$$

It is very natural to assert that the function $F_{a}(x)$ is strictly increasing on $\left(0, \frac{1}{a}\right)$ and it is both strictly increasing and strictly concave on $\left(\frac{1}{a}, \infty\right)$. More strongly, we conjecture that

$$
(-1)^{n-1}\left[F_{a}(x)\right]^{(n)}>0
$$

for $x>\frac{1}{a}$ and $n \in \mathbb{N}$.

Remark 3. From Theorem 3, it is easy to see that the right-hand side inequality in (12) is sharp, but the left-hand side inequality can be sharpened by replacing the constant $\frac{2}{3}=0.666 \cdots$ by a larger number $\frac{3(2 \ln 2-\ln 3) \ln 3}{2 \ln 2}=0.683 \ldots$

Remark 4. We conjecture that

$$
(-1)^{k-1}[G(x)]^{(k)}>0
$$

for $k \in \mathbb{N}$ on $(0, \infty)$, that is, the function $1-G(x)$ is completely monotonic on $(0, \infty)$.

Remark 5. In [17] and its revised version [19], the reciprocal of the function $[\Gamma(x+$ $1)]^{1 / x}$ was proved to be logarithmically completely monotonic on $(-1, \infty)$. Consequently, the function

$$
Q(x)=\left[\frac{\pi^{x / 2}}{\Gamma(1+x / 2)}\right]^{1 / x}=\frac{\sqrt{\pi}}{[\Gamma(1+x / 2)]^{1 / x}}
$$

is also logarithmically completely monotonic on $(-2, \infty)$. In particular, the function $Q(x)$ is both strictly decreasing and strictly logarithmically convex on $(-2, \infty)$. Because $Q(n)=\Omega_{n}^{1 / n}$ for $n \in \mathbb{N}$, the sequence $\Omega_{n}^{1 / n}$ is strictly decreasing and 
strictly logarithmically convex for $n \in \mathbb{N}$. This generalizes one of the results in $[5$, Lemma 2.40] mentioned above.

Furthermore, from the logarithmically complete monotonicity of $Q(x)$, it is easy to obtain that the sequence

$$
\frac{\Omega_{n}^{1 / n}}{\Omega_{n+1}^{1 /(n+1)}}
$$

is also strictly decreasing and strictly logarithmically convex for $n \in \mathbb{N}$. As a direct consequence of the decreasing monotonicity of the sequence (18), the following double inequality may be derived:

$$
\Omega_{n+1}^{n /(n+1)}<\Omega_{n} \leq\left(\frac{2}{\sqrt{\pi}}\right)^{n} \Omega_{n+1}^{n /(n+1)}, \quad n \in \mathbb{N} .
$$

When $1 \leq n \leq 4$, the right-hand side inequality in (19) is better than the corresponding one in

$$
\frac{2}{\sqrt{\pi}} \Omega_{n+1}^{n /(n+1)} \leq \Omega_{n}<\sqrt{e} \Omega_{n+1}^{n /(n+1)}, \quad n \in \mathbb{N}
$$

obtained in [1, Theorem 1].

Remark 6. In [16], the inequality

$$
\frac{[\Gamma(x+y+1) / \Gamma(y+1)]^{1 / x}}{[\Gamma(x+y+2) / \Gamma(y+1)]^{1 /(x+1)}}<\sqrt{\frac{x+y}{x+y+1}}
$$

was confirmed to be valid if and only if $x+y>y+1>0$ and to be reversed if and only if $0<x+y<y+1$. Taking $y=0$ and $x=\frac{n}{2}$ in (21) leads to

$$
\frac{[\Gamma(n / 2+1)]^{1 / n}}{[\Gamma((n+2) / 2+1)]^{1 /(n+2)}}=\frac{\Omega_{n+2}^{1 /(n+2)}}{\Omega_{n}^{1 / n}}<\sqrt[4]{\frac{n}{n+2}}, \quad n>2 .
$$

Similarly, if letting $y=1$ and $x=\frac{n+1}{2}>1$ in (21), then

$$
\frac{\Omega_{n+5}^{1 /(n+3)}}{\Omega_{n+3}^{1 /(n+1)}}<\frac{1}{\pi^{2 /(n+1)(n+3)}} \sqrt[4]{\frac{n+3}{n+5}}, \quad n \geq 2 .
$$

Remark 7. In [18], the following double inequality was discovered: For $t>0$ and $y>-1$, the inequality

$$
\left(\frac{x+y+1}{x+y+t+1}\right)^{a}<\frac{[\Gamma(x+y+1) / \Gamma(y+1)]^{1 / x}}{[\Gamma(x+y+t+1) / \Gamma(y+1)]^{1 /(x+t)}}<\left(\frac{x+y+1}{x+y+t+1}\right)^{b}
$$

holds with respect to $x \in(-y-1, \infty)$ if $a \geq \max \left\{1, \frac{1}{y+1}\right\}$ and $b \leq \min \left\{1, \frac{1}{2(y+1)}\right\}$. Letting $t=1, y=0$ and $x=\frac{n}{2}$ for $n \in \mathbb{N}$ in $(24)$ reveals that

$$
\frac{n+2}{n+4}<\frac{[\Gamma(n / 2+1)]^{2 / n}}{[\Gamma((n+2) / 2+1)]^{2 /(n+2)}}<\sqrt{\frac{n+2}{n+4}}
$$

which is equivalent to

$$
\sqrt{\frac{n+2}{n+4}}<\frac{\Omega_{n+2}^{1 /(n+2)}}{\Omega_{n}^{1 / n}}<\sqrt[4]{\frac{n+2}{n+4}}, \quad n \in \mathbb{N} .
$$

When $n \geq 3$, the inequality (22) is better than the right-hand side inequality in (25). 
If taking $t=1, y=1$ and $x=\frac{n}{2}-1$, then

$$
\frac{1}{\pi^{2 /[(n-2) n]}} \sqrt{\frac{n+2}{n+4}}<\frac{\Omega_{n+2}^{1 / n}}{\Omega_{n}^{1 /(n-2)}}<\frac{1}{\pi^{2 /[(n-2) n]}} \sqrt[8]{\frac{n+2}{n+4}}, \quad n \in \mathbb{N} .
$$

Amazingly, replacing $t$ by $\frac{1}{2}, y$ by 0 , and $x$ by $\frac{n}{2}$ in (24) results in

$$
\sqrt{\frac{n+2}{n+3}}<\frac{\Omega_{n+1}^{1 /(n+1)}}{\Omega_{n}^{1 / n}}<\sqrt[4]{\frac{n+2}{n+3}}
$$

for $n \geq-1$. When $n>2$, this refines the inequality (20) in [1, Theorem 1].

Similarly, by setting different values of $x, y$ and $t$ in inequalities (21) and (24), more similar inequalities as above may be derived immediately.

Remark 8 . Now it is very clear that the inequality (20) obtained in [1, Theorem 1$]$ was thoroughly strengthened by (19) and (27) together.

Remark 9 . The inequality (27) and other related ones derived above motivate us to ask the following question: What are the best positive constants $a \geq 3, b \leq 3$, $\lambda \leq 1, \mu \geq 1, \alpha \geq 2$, and $\beta \leq 4$ such that the inequality

$$
\sqrt[\alpha]{1-\frac{\lambda}{n+a}}<\frac{\Omega_{n+1}^{1 /(n+1)}}{\Omega_{n}^{1 / n}}<\sqrt[\beta]{1-\frac{\mu}{n+b}}
$$

holds true for $n \in \mathbb{N}$ ?

\section{LEMMAS}

In order to prove our theorems, we need the following lemmas.

Lemma 1 ([11, 12, 20]). For $x \in(0, \infty)$ and $k \in \mathbb{N}$, we have

$$
\ln x-\frac{1}{x}<\psi(x)<\ln x-\frac{1}{2 x}
$$

and

$$
\frac{(k-1) !}{x^{k}}+\frac{k !}{2 x^{k+1}}<(-1)^{k+1} \psi^{(k)}(x)<\frac{(k-1) !}{x^{k}}+\frac{k !}{x^{k+1}} .
$$

Lemma 2 ([8, Theorem 2]). For $x>1$, we have

$$
\left(1+\frac{1}{x}\right)^{x}>\frac{x+1}{[\Gamma(x+1)]^{1 / x}} .
$$

The inequality (31) is reversed for $0<x<1$.

Lemma 3 ([15, 21]). If $t>0$, then

$$
\frac{2 t}{2+t}<\ln (1+t)<\frac{t(2+t)}{2(1+t)}
$$

If $-1<t<0$, the inequality (32) is reversed. 


\section{Proofs of TheOREMS}

Proof of Theorem 1. The inequality (7) means that the function (6) is positive and increasing on $(0, \infty)$. It is apparent that the function

$$
\frac{\ln x}{\ln (2 x)}=\frac{1}{1+\ln 2 / \ln x}
$$

is positive and strictly increasing on $\left(0, \frac{1}{2}\right)$ and $(1, \infty)$. Therefore, the function

$$
F(x)=\frac{\ln \Gamma(x+1)}{x \ln x} \cdot \frac{\ln x}{\ln (2 x)}
$$

is strictly increasing on $\left(0, \frac{1}{2}\right)$ and $(1, \infty)$.

The inequality (31) for $0<x<1$ in Lemma 2 can be rewritten as

$$
\frac{\ln \Gamma(x+1)}{x}<(1-x) \ln (x+1)+x \ln x .
$$

Direct calculation yields

$$
F^{\prime}(x)=\frac{x \ln (2 x) \psi(x+1)-[\ln (2 x)+1] \ln \Gamma(x+1)}{x^{2}[\ln (2 x)]^{2}} \triangleq \frac{\theta(x)}{x^{2}[\ln (2 x)]^{2}}
$$

and

$$
\theta^{\prime}(x)=x \ln (2 x) \psi^{\prime}(x+1)-\frac{\ln \Gamma(x+1)}{x} .
$$

Utilizing the left-hand side inequality in (30) for $k=1$, the inequality (34) and Lemma 3 leads to

$$
\begin{aligned}
\theta^{\prime}(x) & >x\left[\frac{1}{x+1}+\frac{1}{2(x+1)^{2}}\right] \ln (2 x)-(1-x) \ln (x+1)-x \ln x \\
& >x\left[\frac{1}{x+1}+\frac{1}{2(x+1)^{2}}\right] \frac{2(2 x-1)}{1+2 x}-\frac{x(1-x)(2+x)}{2(1+x)}-x \ln x \\
& =x\left[\frac{2 x^{4}+5 x^{3}+8 x^{2}+3 x-8}{2(x+1)^{2}(2 x+1)}-\ln x\right] \\
& >x\left[\frac{2 x^{4}+5 x^{3}+8 x^{2}+3 x-8}{2(x+1)^{2}(2 x+1)}-\frac{2(x-1)}{1+x}\right] \\
& =\frac{x\left(2 x^{4}-3 x^{3}+4 x^{2}+11 x-4\right)}{2(x+1)^{2}(2 x+1)} \\
& =\frac{x\left[2 x^{2}(x-1)^{2}+x(x+1)^{2}+10 x-4\right]}{2(x+1)^{2}(2 x+1)} \\
& >0
\end{aligned}
$$

for $\frac{1}{2}<x<1$. Hence, the function $\theta(x)$ is strictly increasing on $\left(\frac{1}{2}, 1\right)$. Since

$$
\theta\left(\frac{1}{2}\right)=-\ln \Gamma\left(\frac{3}{2}\right)=-\left(\frac{1}{2} \ln \pi-\ln 2\right)>0,
$$

the function $\theta(x)$, and so the function $F^{\prime}(x)$, is positive, and then the function $F(x)$ is increasing on $\left(\frac{1}{2}, 1\right)$. In a word, the function $F(x)$ is strictly increasing on $\left(0, \frac{1}{2}\right)$ and $\left(\frac{1}{2}, \infty\right)$.

Ready computation gives 


$$
\begin{aligned}
\frac{x^{3}[\ln (2 x)]^{3} F^{\prime \prime}(x)}{2[\ln (2 x)]^{2}+3 \ln (2 x)+2} & =\ln \Gamma(x+1) \\
+ & \frac{x^{2}[\ln (2 x)]^{2} \psi^{\prime}(x+1)-2 x \ln (2 x)[\ln (2 x)+1] \psi(x+1)}{2[\ln (2 x)]^{2}+3 \ln (2 x)+2}
\end{aligned}
$$

and

$$
\frac{\mathrm{d}}{\mathrm{d} x}\left\{\frac{x^{3}[\ln (2 x)]^{3} F^{\prime \prime}(x)}{2[\ln (2 x)]^{2}+3 \ln (2 x)+2}\right\}=\frac{[\ln (2 x)]^{2} \phi(x)}{\left\{2[\ln (2 x)]^{2}+3 \ln (2 x)+2\right\}^{2}}
$$

for $x>\frac{1}{2}$, where, by making use of the right-hand side inequality in (29) and the inequality (30) for $k=1,2$,

$$
\begin{aligned}
\phi(x)= & {[2 \ln (2 x)+5] \psi(x+1) } \\
& +x\left\{x\left[2[\ln (2 x)]^{2}+3 \ln (2 x)+2\right] \psi^{\prime \prime}(x+1)-[4 \ln (2 x)+3] \psi^{\prime}(x+1)\right\} \\
< & {[2 \ln (2 x)+5]\left[\ln (x+1)-\frac{1}{2(x+1)}\right] } \\
& +x\left\{-x\left[2[\ln (2 x)]^{2}+3 \ln (2 x)+2\right]\left[\frac{1}{(x+1)^{2}}+\frac{1}{(x+1)^{3}}\right]\right. \\
& \left.-[4 \ln (2 x)+3]\left[\frac{1}{x+1}+\frac{1}{2(x+1)^{2}}\right]\right\} \\
= & {[2 \ln (2 x)+5]\left[\ln (x+1)-\frac{1}{2(x+1)}\right] } \\
& -\frac{x\left\{4 x(x+2)[\ln (2 x)]^{2}+2\left(7 x^{2}+16 x+6\right) \ln (2 x)+10 x^{2}+23 x+9\right\}}{2(x+1)^{3}}
\end{aligned}
$$

$\triangleq \varphi(x)$

and

$$
\begin{aligned}
-x(x+1)^{4} \varphi^{\prime}(x)= & 2 x^{4}+10 x^{3}+19 x^{2}+6 x+1+2(x+4) x^{2}[\ln (2 x)]^{2} \\
& +x\left(2 x^{3}+10 x^{2}+20 x+3\right) \ln (2 x)-2(x+1)^{4} \ln (x+1) \\
\triangleq & h(x), \\
h^{\prime}(x)= & 8 x^{3}+34 x^{2}+52 x+7+2 x(3 x+8)[\ln (2 x)]^{2} \\
& +\left(8 x^{3}+34 x^{2}+56 x+3\right) \ln (2 x)-8(x+1)^{3} \ln (x+1), \\
x h^{\prime \prime}(x)= & 24 x^{3}+86 x^{2}+100 x+3+4 x(3 x+4)[\ln (2 x)]^{2} \\
& +8 x\left(3 x^{2}+10 x+11\right) \ln (2 x)-24 x(x+1)^{2} \ln (x+1) \\
\triangleq & q(x), \\
q^{\prime}(x)= & 4\left\{18 x^{2}+57 x+47+(6 x+4)[\ln (2 x)]^{2}\right. \\
& \left.+2\left(9 x^{2}+23 x+15\right) \ln (2 x)-6\left(3 x^{2}+4 x+1\right) \ln (x+1)\right\}, \\
x q^{\prime \prime}(x)= & 4\left\{36 x^{2}+97 x+30+6[\ln (2 x)]^{2} x-12(3 x+2) \ln (x+1) x\right. \\
& \left.+\left(36 x^{2}+58 x+8\right) \ln (2 x)\right\}, \\
& 8\left\{18 x^{3}+53 x^{2}+18 x-11-18(x+1) x^{2} \ln (x+1)\right. \\
& \left.+2\left(9 x^{3}+12 x^{2}+x-2\right) \ln (2 x)\right\} \\
\triangleq & 8 p(x),
\end{aligned}
$$




$$
\begin{aligned}
x p^{\prime}(x)= & 2\left[27 x^{3}+65 x^{2}+10 x-2-9(3 x+2) x^{2} \ln (x+1)\right. \\
& \left.+\left(27 x^{2}+24 x+1\right) x \ln (2 x)\right], \\
(x+1) x^{2} p^{\prime \prime}(x)= & 2\left[54 x^{4}+152 x^{3}+90 x^{2}+3 x+2+6\left(9 x^{2}+13 x\right.\right. \\
& \left.+4) x^{2} \ln (2 x)-18\left(3 x^{2}+4 x+1\right) x^{2} \ln (x+1)\right], \\
(x+1)^{2} x^{3} p^{(3)}(x)= & 2\left[54 x^{5}+168 x^{4}+146 x^{3}+18 x^{2}-9 x-4\right. \\
& \left.+54(x+1)^{2} x^{3} \ln (2 x)-54(x+1)^{2} x^{3} \ln (x+1)\right], \\
(x+1)^{3} x^{4} p^{(4)}(x)= & -4\left(3 x^{5}+8 x^{4}-9 x^{2}-19 x-6\right) \\
& \triangleq-4 \lambda(x), \\
\lambda^{\prime}(x)= & 15 x^{4}+32 x^{3}-18 x-19, \\
\lambda^{\prime \prime}(x)= & 60 x^{3}+96 x^{2}-18 .
\end{aligned}
$$

Since $\lambda^{\prime \prime}(x)$ is strictly increasing for $x>\frac{1}{2}$ and $\lambda^{\prime \prime}\left(\frac{1}{2}\right)=\frac{27}{2}$, the function $\lambda^{\prime \prime}(x)>0$, so $\lambda^{\prime}(x)$ is strictly increasing for $x>\frac{1}{2}$. From $\lambda^{\prime}\left(\frac{1}{2}\right)=-\frac{369}{16}$ and $\lim _{x \rightarrow \infty} \lambda^{\prime}(x)=$ $\infty$, it follows that the function $\lambda^{\prime}(x)$ has a unique zero which is a minimum of $\lambda(x)$ for $x>\frac{1}{2}$. Since $\lambda\left(\frac{1}{2}\right)=-\frac{549}{32}$ and $\lim _{x \rightarrow \infty} \lambda(x)=\infty$, the functions $\lambda(x)$ and $p^{(4)}(x)$ has a unique zero which is a maximum of $p^{(3)}(x)$. Due to $p^{(3)}\left(\frac{1}{2}\right)=$ $188-108 \ln \left(\frac{3}{2}\right)=144.20 \cdots$ and $\lim _{x \rightarrow \infty} p^{(3)}(x)=108(1+\ln 2)$, the function $p^{(3)}(x)$ is positive, thus the function $p^{\prime \prime}(x)$ is strictly increasing for $x>\frac{1}{2}$. Owing to $p^{\prime \prime}\left(\frac{1}{2}\right)=258-90 \ln \left(\frac{3}{2}\right)=221.50 \ldots$, the function $p^{\prime \prime}(x)$ is positive and $p^{\prime}(x)$ is strictly increasing for $x>\frac{1}{2}$. By virtue of $p^{\prime}\left(\frac{1}{2}\right)=\frac{1}{2}\left[181-63 \ln \left(\frac{3}{2}\right)\right]=77.72 \ldots$, it is easy to see that $p^{\prime}(x)>0$ and $p(x)$ is strictly increasing for $x>\frac{1}{2}$. Owing to $p\left(\frac{1}{2}\right)=\frac{27}{4}\left[2-\ln \left(\frac{3}{2}\right)\right]=10.76 \ldots$, it is deduced that $p(x)>0$ and $q^{(3)}(x)>0$ for $x>\frac{1}{2}$, consequently the function $q^{\prime \prime}(x)$ is strictly increasing for $x>\frac{1}{2}$. On account of $q^{\prime \prime}\left(\frac{1}{2}\right)=700-168 \ln \left(\frac{3}{2}\right)=631.88 \ldots$, we obtain that $q^{\prime \prime}(x)>0$ and $q^{\prime}(x)$ is strictly increasing for $x>\frac{1}{2}$. In virtue of $q^{\prime}\left(\frac{1}{2}\right)=320-90 \ln \left(\frac{3}{2}\right)$, we have $q^{\prime}(x)>0$, and then the function $q(x)$ is strictly increasing for $x>\frac{1}{2}$. Because of $q\left(\frac{1}{2}\right)=\frac{155}{2}-27 \ln \left(\frac{3}{2}\right)=66.55 \ldots$, we acquire that $q(x)>0$ and $h^{\prime \prime}(x)>0$, so $h^{\prime}(x)$ is strictly increasing for $x>\frac{1}{2}$. By $h^{\prime}\left(\frac{1}{2}\right)=\frac{85}{2}-27 \ln \left(\frac{3}{2}\right)=33.55 \ldots$, it is derived that $h^{\prime}(x)>0$ and $h(x)$ is strictly increasing for $x>\frac{1}{2}$. Since $h\left(\frac{1}{2}\right)=$ $\frac{81}{8}\left[1-\ln \left(\frac{3}{2}\right)\right]=6.01 \ldots$, we gain that $h(x)>0$ and $\varphi^{\prime}(x)<0$, accordingly $\varphi(x)$ is strictly decreasing for $x>\frac{1}{2}$. Due to $\varphi\left(\frac{1}{2}\right)=-\frac{91}{27}+5 \ln \left(\frac{3}{2}\right)=-1.34 \ldots$, it is procured that $\varphi(x)<0$, i.e., $\phi(x)<0$, hence

$$
\frac{\mathrm{d}}{\mathrm{d} x}\left\{\frac{x^{3}[\ln (2 x)]^{3} F^{\prime \prime}(x)}{2[\ln (2 x)]^{2}+3 \ln (2 x)+2}\right\}<0
$$

and the function

$$
\frac{x^{3}[\ln (2 x)]^{3} F^{\prime \prime}(x)}{2[\ln (2 x)]^{2}+3 \ln (2 x)+2}
$$

is strictly decreasing for $x>\frac{1}{2}$. By

$$
\left.\left\{\frac{x^{3}[\ln (2 x)]^{3} F^{\prime \prime}(x)}{2[\ln (2 x)]^{2}+3 \ln (2 x)+2}\right\}\right|_{x=1 / 2}=\ln \frac{\sqrt{\pi}}{2}=-0.12 \ldots,
$$


it follows that

$$
\frac{x^{3}[\ln (2 x)]^{3} F^{\prime \prime}(x)}{2[\ln (2 x)]^{2}+3 \ln (2 x)+2}<0,
$$

which is equivalent to $F^{\prime \prime}(x)<0$ for $x>\frac{1}{2}$. As a result, the function $F(x)$ is concave on $\left(\frac{1}{2}, \infty\right)$. The proof of Theorem 1 is complete.

Proof of Theorem 2. Let

$$
f(x)=\left[\frac{\pi^{x / 2}}{\Gamma(1+x / 2)}\right]^{1 /(x \ln x)}
$$

for $x>0$. Taking the logarithm of $f(x)$ gives

$$
\ln f(x)=\frac{\ln \pi}{2 \ln x}-\frac{\ln \Gamma(1+x / 2)}{x \ln x}=\frac{\ln \pi}{2 \ln x}-2 F\left(\frac{x}{2}\right),
$$

where $F(x)$ is defined by (8) for $x>0$ and $x \neq \frac{1}{2}$. Differentiating twice gives

$$
[\ln f(x)]^{\prime \prime}=\frac{(\ln x+2) \ln \pi}{2 x^{2}(\ln x)^{3}}-\frac{1}{2} F^{\prime \prime}\left(\frac{x}{2}\right) .
$$

Since $F(x)$ is strictly concave for $x>\frac{1}{2}$, then the function $[\ln f(x)]^{\prime \prime}$ is positive for $x>1$. As a result, the function $f(x)$ is strictly logarithmically convex for $x>1$, and so the sequence $f(n)=\Omega_{n}^{1 /(n \ln n)}$ for $n \geq 2$ is strictly logarithmically convex.

Since the function $f(x)$ is strictly logarithmically convex for $x>1$, then $[\ln f(x)]^{\prime}$ is strictly increasing for $x>1$, therefore

$$
\left[\ln \frac{f(x)}{f(x+1)}\right]^{\prime}=[\ln f(x)-\ln f(x+1)]^{\prime}=[\ln f(x)]^{\prime}-[\ln f(x+1)]^{\prime}<0
$$

for $x>1$. This implies that the function $\frac{f(x)}{f(x+1)}$ is strictly decreasing for $x>1$, hence the sequence $\frac{f(n)}{f(n+1)}$ is also strictly decreasing for $n \geq 2$. The proof of Theorem 2 is thus completed.

Proof of Theorem 3. It is easy to see that the function $G(x)$ in (12) may be rearranged as

$$
G(x)=\frac{\ln x}{\ln (x+1)} \ln \left(1+\frac{1}{x}\right)^{x} .
$$

It is common knowledge that the function $\left(1+\frac{1}{x}\right)^{x}$ is strictly increasing and greater than 1 on $(0, \infty)$ and tends to $e$ as $x \rightarrow \infty$. Furthermore, for $x>0$,

$$
\left[\frac{\ln x}{\ln (x+1)}\right]^{\prime}=\frac{(x+1) \ln (x+1)-x \ln x}{x(x+1)[\ln (x+1)]^{2}}>0 .
$$

Thus, the function $\frac{\ln x}{\ln (x+1)}$ is strictly increasing on $(0, \infty)$ and positive on $(1, \infty)$ and, by L'Hôspital's rule, tends to 1 as $x \rightarrow \infty$. Therefore, the second limit in (13) is valid and the function $G(x)$ is strictly increasing on $(1, \infty)$. The first limit in (13) can be calculated by L'Hôspital's rule as follows

$$
\begin{aligned}
\lim _{x \rightarrow 0^{+}} G(x) & =\lim _{x \rightarrow 0^{+}} \frac{1-\ln x / \ln (x+1)}{1 / x} \lim _{x \rightarrow 0^{+}} \ln x \\
& =\lim _{x \rightarrow 0^{+}} \frac{x[(x+1) \ln (x+1)-x \ln x]}{(x+1)[\ln (x+1)]^{2}} \lim _{x \rightarrow 0^{+}} \ln x
\end{aligned}
$$




$$
\begin{aligned}
& =\lim _{x \rightarrow 0^{+}}\left\{\frac{x}{\ln (x+1)}\left[1-\frac{x \ln x}{(x+1) \ln (x+1)}\right]\right\} \lim _{x \rightarrow 0^{+}} \ln x \\
& =\lim _{x \rightarrow 0^{+}}\left[1-\frac{x}{\ln (x+1)} \cdot \frac{\ln x}{x+1}\right] \lim _{x \rightarrow 0^{+}} \ln x \\
& =-\infty
\end{aligned}
$$

The function $G(x)$ can also be rearranged as

$$
G(x)=\frac{x \ln x}{\ln (x+1)}[\ln (x+1)-\ln x] \triangleq f_{1}(x) f_{2}(x)
$$

for $x \in(0, \infty)$. It is not difficult to see that the function $f_{2}(x)$ is positive and decreasing on $(0, \infty)$. Straightforward differentiation produces

$$
f_{1}^{\prime}(x)=\frac{\ln (x+1)+[\ln (x+1)-x /(x+1)] \ln x}{[\ln (x+1)]^{2}} \triangleq \frac{g(x)}{[\ln (x+1)]^{2}}
$$

and $f_{1}(1)=0$. By virtue of the double inequality (32), it follows that

$$
\begin{aligned}
g(x) & >\frac{2 x}{2+x}+\left[\frac{x(2+x)}{2(1+x)}-\frac{x}{x+1}\right] \ln x \\
& =\frac{2 x}{2+x}+\frac{x^{2}}{2(1+x)} \ln x \\
& =\frac{x^{2}}{2(1+x)}\left[\frac{4(1+x)}{x(2+x)}+\ln x\right] \\
& \triangleq \frac{x^{2}}{2(1+x)} h(x)
\end{aligned}
$$

and

$$
h^{\prime}(x)=\frac{x^{3}-4 x-8}{x^{2}(x+2)^{2}}<0
$$

for $x \in(0,1)$. As a result, the function $h(x)$ is decreasing on $(0,1)$ with $h(1)=\frac{8}{3}$, so $h(x)>0$ on $(0,1)$. This means that the functions $g(x)$ and $f_{1}^{\prime}(x)$ are positive on $(0,1)$. Hence, the function $f_{1}(x)$ is increasing and negative on $(0,1)$. In conclusion, the function $G(x)=f_{1}(x) f_{2}(x)$ is increasing and negative on $(0,1)$. The proof of Theorem 3 is complete.

Second proof of a part of Theorem 3. By (37), it is clear that the function

$$
f_{2}(x)=\int_{x}^{x+1} \frac{1}{t} \mathrm{~d} t=\int_{0}^{1} \frac{1}{t+x} \mathrm{~d} t
$$

is completely monotonic on $(0, \infty)$.

On the other hand, we have

$$
f_{1}(x)=\frac{u(x)-u(0)}{v(x)-v(0)}
$$

and

$$
\frac{u^{\prime}(x)}{v^{\prime}(x)}=(x+1)(1+\ln x)
$$

is increasing on $(0, \infty)$, where

$$
u(x)= \begin{cases}x \ln x, & x \in(0,1] \\ 0, & x=0\end{cases}
$$


and $v(x)=\ln (x+1)$ for $x \in[0,1]$. The monotonic form of L'Hôspital's rule put forward in [4, Theorem 1.25] (or see [14, p. 92, Lemma 1] and [22, p. 10, Lemma 2.9]) reads that if $U$ and $V$ are continuous on $[a, b]$ and differentiable on $(a, b)$ such that $V^{\prime}(x) \neq 0$ and $\frac{U^{\prime}(x)}{V^{\prime}(x)}$ is increasing (or decreasing) on $(a, b)$, then the functions $\frac{U(x)-U(b)}{V(x)-V(b)}$ and $\frac{U(x)-U(a)}{V(x)-V(a)}$ are also increasing (or decreasing) on $(a, b)$. Therefore, the function $f_{1}(x)$ is increasing and negative on $(0,1)$. In a word, the function $G(x)=f_{1}(x) f_{2}(x)$ is increasing and negative on $(0,1)$.

\section{REFERENCES}

[1] H. Alzer, Inequalities for the volume of the unit ball in $\mathbb{R}^{n}$, J. Math. Anal. Appl. 252 (2000), 353-363.

[2] H. Alzer, Inequalities for the volume of the unit ball in $\mathbb{R}^{n}$, II, Mediterr. J. Math. 5 (2008), no. $4,395-413$.

[3] G. D. Anderson and S.-L. Qiu, A monotoneity property of the gamma function, Proc. Amer. Math. Soc. 125 (1997), 3355-3362.

[4] G. D. Anderson, M. K. Vamanamurthy and M. Vuorinen, Conformal Invariants, Inequalities, and Quasi-conformal Maps, John Wiley \& Sons, New York, 1997.

[5] G. D. Anderson, M. K. Vamanamurthy, and M. Vuorinen, Special functions of quasiconformal theory, Expo. Math. 7 (1989), 97-136.

[6] C. Berg and H. L. Pedersen, A completely monotone function related to the gamma function, Proceedings of the Fifth International Symposium on Orthogonal Polynomials, Special Functions and their Applications (Patras, 1999). J. Comput. Appl. Math. 133 (2001), no. 1-2, 219-230.

[7] C. Berg and H. L. Pedersen, Pick functions related to the gamma function, Conference on Special Functions (Tempe, AZ, 2000). Rocky Mountain J. Math. 32 (2002), no. 2, 507-525.

[8] Ch.-P. Chen and F. Qi, Inequalities relating to the gamma function, Austral. J. Math. Anal. Appl. 1 (2004), no. 1, Art. 3; Available online at http://ajmaa.org/cgi-bin/paper.pl? string=v1n1/V1I1P3.tex.

[9] Ch.-P. Chen and F. Qi, Note on a monotonicity property of the gamma function, Octogon Math. Mag. 12 (2004), no. 1, 123-125.

[10] Á. Elbert and A. Laforgia, On some properties of the gamma function, Proc. Amer. Math. Soc. 128 (2000), no. 9, 2667-2673.

[11] B.-N. Guo, R.-J. Chen and F. Qi, A class of completely monotonic functions involving the polygamma functions, J. Math. Anal. Approx. Theory 1 (2006), no. 2, 124-134.

[12] B.-N. Guo and F. Qi, Two new proofs of the complete monotonicity of a function involving the psi function, Bull. Korean Math. Soc. 47 (2010), no. 1, 103-111; Available online at http://dx.doi.org/10.4134/BKMS.2010.47.1.103.

[13] X. Li, Monotonicity properties for the gamma and psi functions, Sci. Magna 4 (2008), no. 4, $18-23$.

[14] D.-W. Niu, J. Cao, and F. Qi, Generalizations of Jordan's inequality and concerned relations, Politehn. Univ. Bucharest Sci. Bull. Ser. A Appl. Math. Phys. 72 (2010), no. 3, 85-98.

[15] F. Qi and Ch.-P. Chen, Monotonicities of two sequences, Mathematics and Informatics Quarterly 9 (1999), no. 4, 136-139.

[16] F. Qi and B.-N. Guo, A complete solution to an open problem relating to an inequality for ratios of gamma functions, Available online at http://arxiv.org/abs/0902.2513.

[17] F. Qi and B.-N. Guo, Complete monotonicities of functions involving the gamma and digamma functions, RGMIA Res. Rep. Coll. 7 (2004), no. 1, Art. 8, 63-72; Available online at http://rgmia.org/v7n1.php.

[18] F. Qi and B.-N. Guo, Necessary and sufficient conditions for a function involving a ratio of gamma functions to be logarithmically completely monotonic, Available online at http: //arxiv.org/abs/0904.1101.

[19] F. Qi and B.-N. Guo, Some logarithmically completely monotonic functions related to the gamma function, J. Korean Math. Soc. 47 (2010), no. 6, 1283-1297; Available online at http://dx.doi.org/10.4134/JKMS.2010.47.6.1283. 
[20] F. Qi, S. Guo and B.-N. Guo, Complete monotonicity of some functions involving polygamma functions, J. Comput. Appl. Math. 233 (2010), no. 9, 2149-2160; Available online at http: $/ / \mathrm{dx}$.doi.org/10.1016/j.cam.2009.09.044.

[21] F. Qi, W. Li, and B.-N. Guo, Generalizations of a theorem of I. Schur, Appl. Math. E-Notes 6 (2006), 244-250.

[22] F. Qi, D.-W. Niu and B.-N. Guo, Refinements, generalizations, and applications of Jordan's inequality and related problems, J. Inequal. Appl. 2009 (2009), Article ID 271923, 52 pages; Available online at http://dx.doi.org/10.1155/2009/271923.

(F. Qi) Department of Mathematics, College of Science, Tianjin Polytechnic UniverSity, Tianjin City, 300160, China

E-mail address: qifeng618@gmail.com, qifeng618@hotmail.com, qifeng618@qq.com

$U R L:$ http://qifeng618.spaces.live.com

(B.-N. Guo) School of Mathematics and Informatics, Henan Polytechnic University, Jiaozuo City, Henan Province, 454010, China

E-mail address: bai.ni.guo@gmail.com, bai.ni.guo@hotmail.com 